\title{
Effects of Aerobic Exercise Based upon Heart Rate at Aerobic Threshold in Obese Elderly Subjects with Type 2 Diabetes
}

\author{
Gian Pietro Emerenziani, ${ }^{1}$ Maria Chiara Gallotta, ${ }^{1}$ Marco Meucci, ${ }^{2}$ Luigi Di Luigi, ${ }^{3}$ \\ Silvia Migliaccio, ${ }^{3}$ Lorenzo Maria Donini, ${ }^{4}$ Felice Strollo, ${ }^{5}$ and Laura Guidetti ${ }^{1}$ \\ ${ }^{1}$ Exercise and Sport Sciences Unit, Department of Movement, Human and Health Sciences, University of Rome "Foro Italico", \\ Piazza Lauro De Bosis 6, 00135 Rome, Italy \\ ${ }^{2}$ Department of Health and Exercise Sciences, Appalachian State University, Boone, USA \\ ${ }^{3}$ Endocrinology Unit, Department of Movement, Human and Health Sciences, University of Rome "Foro Italico", \\ Piazza Lauro De Bosis 6, 00135 Rome, Italy \\ ${ }^{4}$ Medical Physiopathology, Food Science and Endocrinology Section, Department of Experimental Medicine, \\ Food Science and Human Nutrition Research Unit, Sapienza University of Rome, Piazzale Aldo Moro 5, 00185 Rome, Italy \\ ${ }^{5}$ Diabetes Care Unit, St. Spirito Hospital, Lungo Tevere in Saxia 1, 00193 Rome, Italy
}

Correspondence should be addressed to Laura Guidetti; laura.guidetti@uniroma4.it

Received 5 August 2014; Revised 16 September 2014; Accepted 17 September 2014

Academic Editor: Ilias Migdalis

Copyright (C) 2015 Gian Pietro Emerenziani et al. This is an open access article distributed under the Creative Commons Attribution License, which permits unrestricted use, distribution, and reproduction in any medium, provided the original work is properly cited.

\begin{abstract}
In obese diabetic subjects, a correct life style, including diet and physical activity, is part of a correct intervention protocol. Thus, the aim of this study was to evaluate the effects of aerobic training intervention, based on heart rate at aerobic gas exchange threshold $\left(\right.$ Aer $\mathrm{T}_{\mathrm{ge}}$ ), on clinical and physiological parameters in obese elderly subjects with type 2 diabetes (OT2DM). Thirty OT2DM subjects were randomly assigned to an intervention (IG) or control group (CG). The IG performed a supervised aerobic exercise training based on heart rate at Aer $\mathrm{T}_{\mathrm{ge}}$ whereas CG maintained their usual lifestyle. Anthropometric measures, blood analysis, peak oxygen consumption $\left(\dot{\mathrm{V}}_{2 \text { peak }}\right)$, metabolic equivalent $\left(\mathrm{MET}_{\text {peak }}\right)$, work rate $\left(\mathrm{WR}_{\text {peak }}\right)$, and $\mathrm{WR}_{\text {AerTge }}$ were assessed at baseline and after intervention. After training, patients enrolled in the IG had significantly higher $(P<0.001) \dot{V O}_{2 \text { peak }}, \mathrm{MET}_{\text {peak }}, \mathrm{WR}_{\text {peak }}$, and $\mathrm{WR}_{\text {AerTge }}$ and significantly lower $(P<0.005)$ weight, BMI, \%FM, and waist circumference than before intervention. Both IG and CG subjects had lower glycated haemoglobin levels after intervention period. No significant differences were found for all the other parameters between pre- and posttraining and between groups. Aerobic exercise prescription based upon HR at AerT $\mathrm{T}_{\text {ge }}$ could be a valuable physical intervention tool to improve the fitness level and metabolic equilibrium in OT2DM patients.
\end{abstract}

\section{Introduction}

Obesity is a leading risk factor for premature mortality and chronic health hazards such as type 2 diabetes, coronary heart diseases, and hypertension. According to the World Health Organization at least 2.8 million adults die each year as a result of being overweight or obese. In addition, $44 \%$ of the diabetes burden, $23 \%$ of the ischaemic heart disease burden, and 7 to $41 \%$ of certain cancers are also attributable to overweight and obesity. The prevalence of overweight and obesity has increased to epidemic proportions in the industrialized world and it is now dramatically rising in low- and middle-income countries, particularly in urban settings. It is well known that regular physical activity (PA) provides health benefits and it is considered an essential component of primary and secondary prevention for most of metabolicsyndrome related pathologies $[1,2]$. Recent experimental data suggests that subjects who increased their level of PA over time have a decreased mortality rate compared to those who were consistently unfit $[3,4]$.

Despite such evidence, physical inactivity remains a global health problem and its negative effects on health were well documented [5] as well as negative economic consequences [6]. The role of exercise intensity on physical 
training adherence is supported by several large studies [7-9]. Furthermore, Dishman and Buckworth [10] showed that activity-promotion efforts were more effective when the intensity was low rather than high. Accordingly, exercise intensity should be set in order to reach positive physiological effects, decrease the risk of injury, and increase adherence. It is well known that exercise can elicit different physiological responses in obese individuals when prescribed in absolute terms [11], since, usually, no adjustment is usually made for each individual's exercise capacity. Moreover, the use of relative terms, such as $\% \mathrm{VO}_{2 \max }$, has been substantially criticized [12-14], since it seems that the relative parameters alone without considering the aerobic threshold (AerT) are not enough to individualize the exercise intensity [12]. AerT is the point after which ventilation begins to increase disproportionately relative to oxygen uptake and it is considered a useful parameter for optimal aerobic exercise intensity prescription in DM2 patients [12, 15-17]. To clarify this specific terminology, Meyer et al. [14] suggested using the terms Aer $_{\text {ge }}$ when aerobic threshold is evaluated by gas analysis.

Moreover, during the last years, Aer $_{\text {ge }}$ has been more frequently used to prescribe exercise intensity in obese and diabetic populations $[12,14,15,18,19]$. Interestingly, there are two different methods to exercise at a relative Aer $\mathrm{T}_{\mathrm{ge}}$ : the first aims at maintaining a work load, such as speed and power, corresponding to AerT, and the second one is keeping a constant HR corresponding to Aer $\mathrm{T}_{\text {ge }}$ [20]. However, since musculoskeletal complications commonly present in diabetic patients [21] could prevent obese elderly patients from performing $30 \mathrm{~min}$ exercise at constant average load, it could be useful to choose an approach based on constant HR at $\mathrm{Aer}_{\mathrm{ge}}$.

The effects of 30 min exercise at constant HR on physiological parameters were previously described by Kindermann et al. [20] who showed that it is possible to maintain the optimal individual workload intensity by regulating HR during exercise. The intensity of PA has been found to be negatively related to adherence in overweight or obese subjects [22]. Moreover, DaSilva et al. [22] showed that overweight individuals choose to exercise at intensities below or around the ventilatory threshold and that this intensity results in low perceived exertion and positive affective responses. Also, frequent walking of sufficient duration performed more than 3 days a week might improve glycaemic control and lipoprotein profiles of subjects with type 2 diabetes and, also, cardiorespiratory fitness $[15,23]$.

To the best of our knowledge, only one study [23] evaluated the effects of heart rate intensity prescribed walking training program on cardiorespiratory fitness and glycaemic control in individuals affected by type 2 diabetes mellitus. Subjects' peak heart rates, obtained from the Balke-Ware protocol, were used to set a training intensity at the $80 \%$ $\mathrm{HR}_{\text {peak }}$ and only walking exercise was performed for a training period of 7 weeks.

Therefore, the aim of our study was to evaluate the effects of three months of aerobic exercise, based upon heart rate at Aer $_{\text {ge }}$ on glycemic control, body weight, and fitness in obese elderly subjects with type 2 diabetes.

\section{Methods}

2.1. Participants. Thirty obese elderly subjects (age $66.8 \pm$ 6.3 years), body mass index (BMI) of $34.6 \pm 3.2 \mathrm{~kg} / \mathrm{m}^{2}$, and percent fat mass (\%FM) of $36.5 \pm 6.8 \%$ with type 2 diabetes, were recruited by a diabetes care unit hospital. Participants provided written informed consent and protocol was approved by the Local Scientific Committee. All subjects were sedentary and they had not been previously engaged in regular physical exercise program. Diagnosis of Type 2 diabetes mellitus (T2DM) was established according to the World Health Organization criteria [24]. Subjects underwent clinical examination to rule out any contraindications to PA such as neuropathy, autonomic dysfunction, cardiovascular diseases, and high blood pressure $(\geq 140 / 90 \mathrm{mmHg})$. All subjects were on oral pharmacological treatments.

2.2. Experimental Design. Subjects were randomly assigned to an intervention group (IG) (15 subjects, age $66.7 \pm 4.9$ years) or to a control group (CG) (15 subjects, age $66.9 \pm 4.2$ years). Pulmonary function tests and a resting electrocardiogram (ECG) recording were performed as initial screening in both groups. Prior to the first test session, participants took part in a familiarization session to become accustomed to PA tests. Anthropometric measurements, blood sampling, dietary and physiological evaluation, and a submaximal graded exercise test were performed at baseline and after 3 months in both groups. During the 3-month intervention period, IG group performed supervised aerobic exercise training while CG did not perform any organized exercise. Lifestyle and food behaviour of the subjects have not changed during the experimental procedure.

2.3. Body Composition. Participant's height was measured at the time of hospital referral using a stadiometer to the nearest $0.1 \mathrm{~cm}$. Body composition was determined using a multifrequency bioimpedance analysis (InBody 720, Biospace Inc., Cerritos, CA, USA) [25]. All subjects were tested in the morning after 12-hour overnight fast. All body mass (BM) measurements were taken on a calibrated digital scale (InBody 720, Biospace Inc., Cerritos, CA, USA) when the subjects wore minimal clothing (i.e., underwear). Body mass index (BMI) was calculated as body mass divided by squared height $\left(\mathrm{kg} / \mathrm{m}^{2}\right)$.

Waist circumferences were taken at $2.5 \mathrm{~cm}$ above the umbilicus [26] using an inextensible metallic tape placed directly on the skin, perpendicularly to the long axis of the body, and horizontally to the floor at the end of normal expiration. Average values from two measurements were considered.

2.4. Blood Analysis. All subjects had $5 \mathrm{~mL}$ blood drawn into a vacutainer from an antecubital vein in the morning after the overnight fast. Serum obtained after clotting and centrifugation at $1500 \mathrm{~g}$ for $20 \mathrm{~min}$ was used for glucose, triglyceride, and total/HDL cholesterol determination by a Kodak Autoanalyzer. Low density lipoprotein cholesterol (LDLC) was determined using Friedwald's equation for 
triglycerides $<400 \mathrm{mg} / \mathrm{dL}$. Additional $2 \mathrm{~mL}$ blood samples were also drawn into EDTA-treated vacutainers for glycated hemoglobin (HbAlc) determination using high-performance liquid chromatography (BioRad Dia-STAT Analyzer 1).

2.5. Aerobic Power. $\dot{\mathrm{V}} \mathrm{O}_{2 \text { peak }}$ was assessed in all participants by means of a continuous, maximal graded exercise test on a cycle ergometer or on a treadmill according to the individual abilities. In particular, subjects who were able to walk safely performed a modified Balke protocol [27] on a treadmill (Run Med Excite, Technogym, Gambettola (FC), Italy), while those that were not able to walk safely performed a bike-ramp 10w protocol [28] on a cycle ergometer (Bike Med Excite, Technogym, Gambettola (FC), Italy). Heart rate (beats. $\mathrm{min}^{-1}$ ) was continuously recorded before and throughout the trial using a HR monitor (RS 400, Polar Electro, Kempele, Finland). $\dot{\mathrm{VO}}_{2}$ and pulmonary ventilation (VE) were measured by a semiportable gas analysis system (Fitmate Pro Cosmed, Rome, Italy) [29]. Prior to each test the Fitmate Pro underwent an automatic gas calibration cycle and the turbine flow meter was periodically calibrated using a $3 \mathrm{~L}$ syringe according to the manufacturer's recommendations. During the test, the highest $\mathrm{VO}_{2}$ attained was chosen as the peak oxygen uptake $\left(\mathrm{V}_{2} \mathrm{O}_{2 \text { peak }}\right)$. The individual aerobic gas exchange threshold $\left(\right.$ Aer $_{\mathrm{ge}}$ ) was determined offline for each subject by plotting the ventilatory equivalent $\left(\dot{\mathrm{VE}} / \mathrm{V}_{2}\right)$ as a function of $\mathrm{VO}_{2}$ to identify the point during exercise where this curve reached its lowest value $[17,30]$. The level of $\mathrm{VO}_{2}$ at which we observed the lowest value of the $\dot{\mathrm{V} E} / \mathrm{V}_{2}$, in the individual plot, was the individual aerobic threshold [12, 31]. Moreover, work rate (WR) and metabolic equivalent (MET) were calculated at AerT $\mathrm{ge}_{\mathrm{ge}}\left(\mathrm{WR}_{\text {AerTge }}\right.$ and $\mathrm{MET}_{\text {AerTge }}$, resp.) and at maximal effort $\left(\mathrm{WR}_{\text {peak }}\right.$ and $\mathrm{MET}_{\text {peak }}$, resp.).

2.6. Training Protocol. Subjects of the IG group performed a 3-month aerobic training (AT) twice a week based on $\mathrm{HR}$ corresponding to their individual Aer $\mathrm{T}_{\text {ge }}$. All training sessions, lasting $50 \mathrm{~min}$, were supervised by a PA specialist. The AT was performed on a treadmill (Run Med Excite, Technogym, Gambettola (FC), Italy) or on a cycle ergometer (Bike Med Excite, Technogym, Gambettola (FC), Italy) in accordance with the device used for the evaluation of $\dot{\mathrm{V}}_{2 \text { peak }}$. Subjects' HR, corresponding to AerT $\mathrm{T}_{\text {ge }}$ obtained from maximal exercise test, was used to set the intensity of training protocol which consisted of a 5 min warm-up, 30 min AT, and 5 min cooldown. All devices were programmed at the beginning of the training to change the external work load (inclination for the treadmill and wattage for the cycle ergometer) to maintain the subjects' HR below (warm-up and cool-down periods) or equal to (training period) the individual subject's $\mathrm{HR}$ at AerT $_{\mathrm{ge}}$. Moreover, the maximal treadmill speed was set not higher than $5 \mathrm{~km} / \mathrm{h}$ to perform all training sessions as safely as possible. Subjects training on a cycle ergometer maintained the pedaling rate at 40 RPM. This pedaling rate was the same used during the incremental graded test. Stretching $(5 \mathrm{~min})$ exercises, involving main lower limb muscle groups, were performed after warm-up and cool-down periods.
2.7. Dietary and Psychological Counselling. Dietary and psychological counselling was performed by a dietitian and a psychologist, respectively, to minimize these items' variability between groups. A low-calorie diet was set at approximately $400 \mathrm{Kcal}$ less than total daily energy expenditure in both IG and CG groups. Total daily energy expenditure was set according to the following equation: resting metabolic rate + physical activity level. Resting metabolic rate was estimated by the Harris benedict equation [32] while physical activity level was estimated by the international physical activity questionnaire (IPAQ) [33]

2.8. Statistical Analysis. The similar baseline characteristics of the two groups were verified by unpaired $t$-test at T0. Due to the between subjects variability of glycated haemoglobin at baseline (T0), a $2 \times 2$ mixed analysis of covariance (ANCOVA) with group (IG versus CG) as between factor, time (before versus after) as within factor, and glycated haemoglobin baseline data as covariate was performed.

For each variable, mixed ANOVA with repeated measures on time was used to detect significant effects of two main factors: group (intervention versus control) and time (before versus after). Post hoc analysis of significant differences for group factor was performed using the unpaired Student's $t$ test, while for time factor it was performed using the paired $t$-test. All statistical analyses were performed with the SPSS statistical package (Version 20.0 for Windows; SPSS Inc., Chicago, IL, USA). All tests were two-tailed, with $\alpha \leq 0.05$ being taken as significant.

\section{Results}

The baseline characteristics were similar (no significant differences) between intervention and control groups for any studied variable as depicted in Table 1 . Subjects in the IG reported a transitory, low muscle pain during training. A significant group $\times$ time interaction $(P<0.05)$ was found for body mass index (BMI), \%FM, and abdominal circumference, indicating that the trend of these variables was different between groups after 3 months. In fact, the aerobic training based upon HR resulted in a significant decrease in $\mathrm{BMI}, \% \mathrm{FM}$, and abdominal circumference, while there were not any differences in CG on anthropometric variables (Table 1). Moreover, significant group $\times$ time interaction $(P<0.05)$ was found for $\dot{V}_{2 \text { peak }}, \mathrm{MET}_{\text {peak }}, \mathrm{WR}_{\text {peak }}$, and $\mathrm{WR}_{\text {AerTge }}$, indicating that only in IG group these variables increased $(P<0.005)$ in posttraining (Table 2$)$. A significant main effect of time was found in glycated haemoglobin that significantly lowered after 3 months in both groups (Figure 1) but no significant effects were found in total cholesterol, high density lipoprotein cholesterol (HDLC), and low density lipoprotein cholesterol (LDLC).

\section{Discussion}

The results presented herein demonstrate that individually designed exercise, at a relative Aer $\mathrm{T}_{\mathrm{ge}}$, improves fitness and metabolic parameters in untrained, sedentary, and obese 
TABLE 1: Anthropometric measures, lipid profile, and glycated haemoglobin for the intervention group (IG) and the control group (CG) at baseline and after 3-month period.

\begin{tabular}{|c|c|c|c|c|}
\hline & \multicolumn{2}{|c|}{ IG $(n=15)$} & \multicolumn{2}{|c|}{ CG $(n=15)$} \\
\hline & T0 & $\mathrm{T} 3$ & T0 & T3 \\
\hline Weight (kg) & $87.6 \pm 19.5$ & $85.0 \pm 17.8^{*}$ & $87.0 \pm 22.6$ & $87.0 \pm 22.1$ \\
\hline $\operatorname{BMI}\left(\mathrm{kg} / \mathrm{m}^{2}\right)$ & $33.6 \pm 7.7$ & $32.6 \pm 7.1^{*}$ & $33.0 \pm 5.4$ & $32.9 \pm 5.3$ \\
\hline Fat mass (\%) & $31.5 \pm 10.4$ & $29.5 \pm 9.5^{*}$ & $32.5 \pm 4.2$ & $31.1 \pm 7.1$ \\
\hline Abdominal circumference $(\mathrm{cm})$ & $117.8 \pm 17.5$ & $115.6 \pm 15.9^{*}$ & $107.0 \pm 12.2$ & $105.4 \pm 12.8$ \\
\hline Glycated haemoglobin $(\mathrm{mmol} / \mathrm{mol})$ & $49.9 \pm 8.3$ & $45.3 \pm 7.3^{*}$ & $53.0 \pm 12.2$ & $45.0 \pm 5.6^{*}$ \\
\hline Total cholesterol (mg/dL) & $210.6 \pm 57.9$ & $217.1 \pm 56.9$ & $163.8 \pm 33.1$ & $162.0 \pm 37.6$ \\
\hline $\operatorname{HDLC}(\mathrm{mg} / \mathrm{dL})$ & $46.6 \pm 16.2$ & $48.7 \pm 13.2$ & $43.8 \pm 12.9$ & $46.7 \pm 13.0$ \\
\hline LDLC (mg/dL) & $122.9 \pm 51.6$ & $136.3 \pm 40.1$ & $96.0 \pm 51.2$ & $99.3 \pm 22.6$ \\
\hline
\end{tabular}

BMI: body mass index; HDLC: high density lipoprotein cholesterol; LDLC: low density lipoprotein cholesterol.

${ }^{*} \mathrm{P}<0.05$ versus $\mathrm{T} 0$.

TABLE 2: Physiological parameters for the intervention group (IG) and the control group (CG) at baseline and after 3-month period.

\begin{tabular}{|c|c|c|c|c|}
\hline & \multicolumn{2}{|c|}{ IG $(n=15)$} & \multicolumn{2}{|c|}{ CG $(n=15)$} \\
\hline & T0 & T3 & T0 & T3 \\
\hline$\overline{\mathrm{V}} \mathrm{O}_{2 \text { peak }}\left(\mathrm{ml} \cdot \mathrm{kg}^{-1} \mathrm{~min}^{-1}\right)$ & $15.9 \pm 3.0$ & $18.5 \pm 3.2^{*}$ & $18.6 \pm 4.2$ & $17.9 \pm 5.7$ \\
\hline $\mathrm{MET}_{\text {peak }}$ & $4.5 \pm 0.8$ & $5.3 \pm 1^{*}$ & $5.3 \pm 1.2$ & $5.1 \pm 1.6$ \\
\hline $\mathrm{WR}_{\text {peak }}(\mathrm{W})$ & $65.7 \pm 25.9$ & $78.6 \pm 26.1^{*}$ & $96.0 \pm 23.0$ & $88.3 \pm 20.0$ \\
\hline$\% \mathrm{HR}_{\max }(\%)$ & $77.1 \pm 9.5$ & $77.9 \pm 7.9$ & $79.0 \pm 5.8$ & $80.3 \pm 7.5$ \\
\hline$\% \dot{\mathrm{V}} \mathrm{O}_{2 \text { peak }}$ at $\mathrm{AerT}_{\mathrm{ge}}(\%)$ & $57.7 \pm 11.9$ & $51.7 \pm 11.6$ & $58.1 \pm 12.1$ & $59.9 \pm 16.5$ \\
\hline$\% \mathrm{HR}_{\max }$ at $\mathrm{AerT}_{\mathrm{ge}}(\%)$ & $57.7 \pm 8.3$ & $56.1 \pm 8.0$ & $60.0 \pm 7.6$ & $60.0 \pm 6.7$ \\
\hline$\%$ HRR at $\operatorname{AerT}_{\text {ge }}(\%)$ & $24.4 \pm 12.2$ & $21.6 \pm 9.8$ & $18.9 \pm 4.7$ & $18.4 \pm 7.2$ \\
\hline WR at $\operatorname{AerT}_{\mathrm{ge}}(\mathrm{w})$ & $20.4 \pm 4.7$ & $28.0 \pm 8.2^{*}$ & $20.8 \pm 9.5$ & $23.3 \pm 0.3$ \\
\hline$\Delta \mathrm{HR}(\mathrm{bpm})$ & $21.5 \pm 11.6$ & $18.9 \pm 9.5$ & $14.2 \pm 3.9$ & $14.3 \pm 7.7$ \\
\hline MET at AerT $_{\mathrm{ge}}$ & $2.6 \pm 0.6$ & $2.6 \pm 0.6$ & $3.1 \pm 0.6$ & $2.9 \pm 0.6$ \\
\hline
\end{tabular}

$\mathrm{VO}_{2 \text { peak }}$ : peak oxygen uptake; MET: metabolic equivalent; $\mathrm{WR}_{\text {peak }}$ : peak work rate.

$\mathrm{HR}_{\max }:$ maximum heart rate; $\mathrm{HRR}$ : heart rate reserve; $\triangle \mathrm{HR}$ : heart rate at $\mathrm{AerT}_{\mathrm{ge}}$-heart rate at rest.

${ }^{*} P<0.05$ versus T0.

diabetic subjects. These positive effects were controlled using baseline glycated haemoglobin as covariate.

Indeed, the first aim of this study was to determine the effects of three months of aerobic exercise training, based on heart rate at aerobic threshold, on long term glycaemic control, body composition, and exercise capacity in OT2DM. In our study, all type 2 diabetic subjects were obese and they did not practice any organized physical activity prior to this study. Moreover, subjects' fitness parameters, such as $\dot{\mathrm{V}}_{2 \text { peak }}$, showed that they were very unfit. According to these observations, we chose not to use high-intensity exercise but a constant moderate intensity exercise as training method. American College of Sports Medicine PA guidelines for type 2 diabetic subjects recommend to perform lowto-moderate intensity physical activity (at $40-70 \% \dot{\mathrm{V}}_{2 \max }$ ) to achieve cardiorespiratory and metabolic improvements. Most importantly, the lower intensity activity affords a more comfortable level of exertion and enhances the likelihood of adherence, while lessening the likelihood of musculoskeletal injury and foot trauma, particularly when weight-bearing activity is recommended [34]. At present, American College of Sports Medicine suggests using three variables to monitor exercise intensity: $\dot{\mathrm{VO}}_{2 \max }, \mathrm{HR}$, and rate of perceived exertion (RPE). Moreover, during the last years, the gas exchange threshold was identified as a valid tool to delineate the "training zone" for endurance training [14] and for unhealthy subjects [12].

In our exercise protocol, subjects performed $30 \mathrm{~min}$ aerobic exercise at an intensity corresponding to their Aer $\mathrm{T}_{\mathrm{ge}}$. In detail, the heart rate determined at Aer $_{\text {ge }}$ was kept constant while the external work load, such as treadmill speed or cycle ergometer watt, decreased in accordance with HR. Kindermann et al. [20] studied the physiological responses of a constant exercise performed at a heart rate corresponding to the anaerobic threshold $(4 \mathrm{mmol} / \mathrm{L})$. In accordance with our results they showed that treadmill speed must be reduced continuously to maintain the HR constant. In our study, subjects who exercised on a cycle ergometer finished the aerobic program with a work load lower than that set at the beginning of training (decrement range 5-20 watts). In accordance, subjects who trained on a treadmill finished the aerobic program with a lower inclination than that at the beginning of training (decrement range $1-4^{\circ}$ ). The decrease in external work load was chosen to allow subjects to perform all 


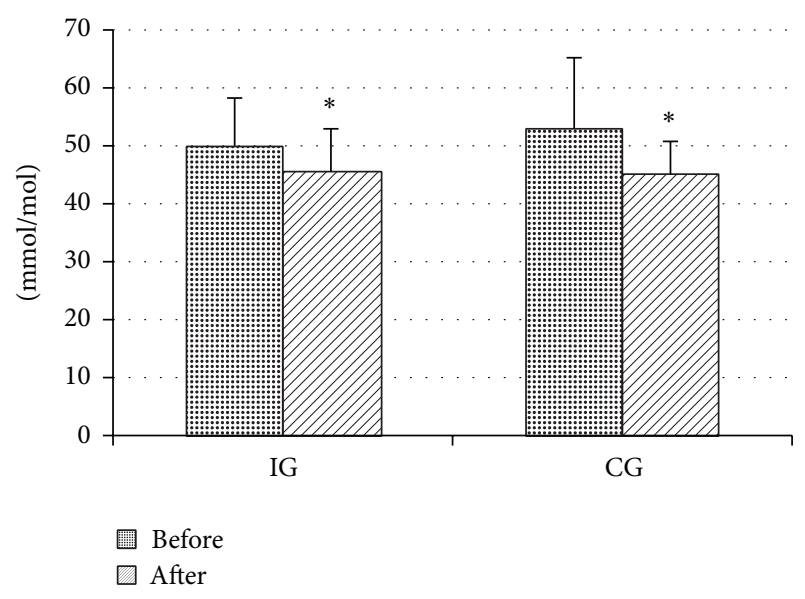

Figure 1: Glycated hemoglobin (HbAlc) before and after 3-month intervention period in control (CG) and intervention groups (IG).

30 min aerobic training because we originally noticed that our obese elderly type 2 diabetic patients were not able to exercise for $30 \mathrm{~min}$ at a constant external work load corresponding to AerT $_{\text {ge }}$.

It is well known that aerobic training associated with resistance exercise might improve glycemic control to an extent comparable to some oral hypoglycemic agents $[35,36]$. The reduction of blood glucose in type 2 diabetic subjects could be reached by either exercising at constant moderate intensity or performing brief high-intensity exercise [15, 37]. Moreover, no data are available regarding the effects of aerobic training based upon constant heart rate at Aer $_{\text {ge }}$ on glycemic control and physiological parameters in diabetes subjects. The reduction in glycated haemoglobin observed in IG $(-9.2 \%)$ was similar to that found in other studies. For example, Belli et al. [15] found that after a 12-week supervised walking training at Aer $_{\text {ge }}$, glycated haemoglobin decreased by $11,6 \%$. Moreover, other two studies, Walker et al. [38] and Shenoy et al. [39], showed a decrease of glycated haemoglobin ranging from $7.6 \%$ to $9.7 \%$, respectively. Contrary to our results, Morton et al. [23] showed no effects on glycated haemoglobin after 7 weeks of heart rate prescribed walking training. The difference between our results and those reported by Morton could be explained by the different duration of the two intervention periods. In fact, as suggested by Kilpatrick [40], glycation of haemoglobin is dependent on mean blood glucose concentrations over the 120-day lifespan of red blood cells. The lower glycated haemoglobin in CG at the end of the study than at baseline could be explained by the fact that all subjects were recruited from local health facilities and they were under pharmacological treatment since the beginning of the study. Therefore, our training protocol did not modify the positive effect on glycated haemoglobin of pharmacological treatment that was equal for both groups.

No significant differences were found in high density lipoprotein cholesterol (HDLC) and low density lipoprotein cholesterol (LDLC) levels in both groups after the training period. This was an unexpected result considering that all subjects received a low-calorie diet at baseline and that the positive effects of diet on lipid profile and weight are well known [41]. Clearly, dieting strategies provide benefits only in complying patients [42]. Indeed, a restricted diet was given at baseline to all subjects, but it was not possible to evaluate adherence to nutritional protocol throughout the study, which might lead to the hypothesis that subjects were not fully compliant to the diet intervention, as also suggested by the small, but significant decrease in body weight, fat mass, and abdominal circumference in IG group.

These results could be explained by the positive effects of physical exercise on energy expenditure. In fact, even if diet adherence was not controlled, IG performed a supervised physical training protocol leading to an energy expenditure increase during the study period.

To our knowledge the aerobic training of the subjects of our study could not be compared with any other study in the literature. In fact, to allow participants to perform a $30 \mathrm{~min}$ aerobic exercise we chose to maintain constant the heart rate corresponding to AerT $_{\text {ge }}$ while the external work load decreased automatically. This method was chosen for two main reasons: firstly, untrained obese older T2DM subjects were very unfit and therefore they could not perform $30 \mathrm{~min}$ exercise at the constant work load corresponding to AerT $_{\text {ge }}$; secondly, the right balance between exercise duration and intensity is a challenging task, since both factors have the potential to negatively impact adherence. PA intensity has been found to be negatively related to adherence in several studies involving overweight participants [4345]. In fact, on average, overweight individuals choose an intensity below or around the AerT ge during a $20 \mathrm{~min}$ bout exercise [22]. Self-paced exercise was reported to fall within the zone of fat maximal oxidation [45] and, when performed over ground, resulted in lower perceived exertion and more positive affective responses than on treadmill [7].

Our results showed that $30 \mathrm{~min}$ aerobic exercise based upon $\mathrm{HR}$ corresponding to AerT $_{\text {ge }}$ could improve physical exercise capacity in obese T2DM subjects as demonstrated by $\dot{\mathrm{VO}}_{2 \text { peak }}, \mathrm{MET}_{\text {peak }}$, and $\mathrm{WR}_{\text {peak }}$ increase after the training period in IG. These findings are in agreement with previous studies even if a different methodology of exercise prescription was applied due to the different characteristics of patients (obese and elderly). For instance, it is well known that when exercise is prescribed referring to absolute parameters (velocity or watt) corresponding to Aer $\mathrm{T}_{\text {ge }}$, exercise capacity can improve in obese adult T2DM $[15,38]$. In addition, Morton et al. showed that walking at a heart rate of $80 \%$ $\mathrm{HR}_{\text {peak }}$, type 2 diabetic subjects improved peak and submaximal cardiorespiratory responses. However, the AerT $\mathrm{T}_{\text {ge }}$ of our subjects was lower than the patients evaluated in other studies $\left(80 \% \mathrm{HR}_{\text {peak }}\right)$. Our positive results support the hypothesis that the lower exercise intensity was balanced by the longer training period used in our protocol.

Moreover the workload corresponding to AerT $\mathrm{T}_{\mathrm{ge}}$ improved after training. In support of this, also Larose et al. [46] observed that workload at AerT $\mathrm{T}_{\text {ge }}$ increased while $\% \dot{\mathrm{VO}_{2 p e a k}}$ at $\mathrm{AerT}_{\text {ge }}$ remained unchanged after 6 months of walking and cycling exercise at $60-75 \% \mathrm{HR}_{\max }$ 
for $25-45 \mathrm{~min}$, three times a week in adult type 2 diabetic subjects.

In conclusion, a 3-month aerobic exercise training based on $\mathrm{HR}$, corresponding to subjects' Aer $_{\text {ge }}$, improved maximal exercise capacity and had positive effects on glycated haemoglobin levels. Thus, the prescription of exercise intensity PA, according to Aer $\mathrm{T}_{\text {ge }}$, should become more frequent in obese diabetic populations, since training at this intensity improves aerobic capacity, cost effectiveness of treatment and could also increase adherence to physical activity in obese subjects.

\section{Conflict of Interests}

The authors declare no conflict of interests.

\section{Acknowledgments}

Research was funded by PRIN 2009-2009KENS9K_004 to Laura Guidetti and Lorenzo Maria Donini and PRIN 2011 052013 to Silvia Migliaccio. Gian Pietro Emerenziani is supported by a fellowship funded by PRIN (year 20092009KENS9K_004). Gian Pietro Emerenziani designed the study, evaluated physical activity of patients, contributed to the interpretation, contributed to the discussion, and wrote the paper; Marco Meucci, Maria Chiara Gallotta evaluated physical activity in patients, analyzed data, contributed to the interpretation and discussion, and reviewed the paper; Luigi Di Luigi contributed to the discussion and reviewed the paper; Lorenzo Maria Donini contributed to interpretation and discussion and reviewed the paper; Silvia Migliaccio recruited and evaluated the patients, analyzed the data, contributed to the interpretation, and wrote the paper; Laura Guidetti designed the study, analyzed the data, contributed to the interpretation, and wrote the paper.

\section{References}

[1] J. E. Donnelly, S. N. Blair, J. M. Jakicic, M. M. Manore, J. W. Rankin, and B. K. Smith, "Appropriate physical activity intervention strategies for weight loss and prevention of weight regain for adults," Medicine and Science in Sports and Exercise, vol. 41, no. 2, pp. 459-471, 2009.

[2] C. E. Garber, B. Blissmer, M. R. Deschenes et al., "Quantity and quality of exercise for developing and maintaining cardiorespiratory, musculoskeletal, and neuromotor fitness in apparently healthy adults: Guidance for prescribing exercise," Medicine and Science in Sports and Exercise, vol. 43, no. 7, pp. 1334-1359, 2011.

[3] L. M. León-Muñoz, D. Martínez-Gómez, T. Balboa-Castillo, E. López-García, P. Guallar-Castillón, and F. Rodríguez-Artalejo, "Continued sedentariness, change in sitting time, and mortality in older adults," Medicine and Science in Sports and Exercise, vol. 45, no. 8, pp. 1501-1507, 2013.

[4] Q.-L. Xue, K. Bandeen-Roche, T. J. Mielenz et al., "Patterns of 12year change in physical activity levels in community-dwelling older women: can modest levels of physical activity help older women live longer?" American Journal of Epidemiology, vol. 176, no. 6 , pp. 534-543, 2012.
[5] F. W. Booth and S. J. Lees, "Fundamental questions about genes, inactivity, and chronic diseases," Physiological Genomics, vol. 28, no. 2, pp. 146-157, 2007.

[6] G. A. Colditz, "Economic costs of obesity and inactivity," Medicine and Science in Sports and Exercise, vol. 31, no. 11, pp. S663-S667, 1999.

[7] S. G. Dasilva, L. Guidetti, C. F. Buzzachera et al., "Psychophysiological responses to self-paced treadmill and overground exercise," Medicine and Science in Sports and Exercise, vol. 43, no. 6, pp. 1114-1124, 2011.

[8] P. Ekkekakis, G. Parfitt, and S. J. Petruzzello, "The pleasure and displeasure people feel when they exercise at different intensities: decennial update and progress towards a tripartite rationale for exercise intensity prescription," Sports Medicine, vol. 41, no. 8, pp. 641-671, 2011.

[9] E. Lind, P. Ekkekakis, and S. Vazou, "The affective impact of exercise intensity that slightly exceeds the preferred level: 'Pain' for no additional 'gain,' Journal of Health Psychology, vol. 13, no. 4, pp. 464-468, 2008.

[10] R. K. Dishman and J. Buckworth, "Increasing physical activity: a quantitative synthesis," Medicine and Science in Sports and Exercise, vol. 28, no. 6, pp. 706-719, 1996.

[11] B. M. Pinet, D. Prud'homme, C. A. Gallant, and P. Boulay, "Exercise intensity prescription in obese individuals," Obesity, vol. 16, no. 9, pp. 2088-2095, 2008.

[12] G. P. Emerenziani, S. Migliaccio, M. C. Gallotta et al., "Physical exercise intensity prescription to improve health and fitness in overweight and obese subjects: a review of the literature," Health, vol. 5, no. 6, pp. 113-121, 2013.

[13] T. Meyer, H. H. W. Gabriel, and W. Kindermann, "Is determination of exercise intensities as percentages of $\dot{\mathrm{VO}} 2$ max or HR(max) adequate?" Medicine and Science in Sports and Exercise, vol. 31, no. 9, pp. 1342-1345, 1999.

[14] T. Meyer, A. Lucía, C. P. Earnest, and W. Kindermann, "A conceptual framework for performance diagnosis and training prescription from submaximal gas exchange parameters-theory and application," International Journal of Sports Medicine, vol. 26, no. 1, pp. S38-S48, 2005.

[15] T. Belli, L. F. P. Ribeiro, M. A. Ackermann, V. Baldissera, C. A. Gobatto, and R. Galdino da Silva, "Effects of 12-week overground walking training at ventilatory threshold velocity in type 2 diabetic women," Diabetes Research and Clinical Practice, vol. 93, no. 3, pp. 337-343, 2011.

[16] J.-F. Brun, S. Bordenave, J. Mercier, A. Jaussent, M.-C. Picot, and C. Préfaut, "Cost-sparing effect of twice-weekly targeted endurance training in type 2 diabetics: a one-year controlled randomized trial," Diabetes \& Metabolism, vol. 34, no. 3, pp. 258-265, 2008.

[17] W. Hollmann, "42 Years ago-development of the concepts of ventilatory and lactate threshold," Sports Medicine, vol. 31, no. 5, pp. 315-320, 2001.

[18] T. G. Babb, B. L. Wyrick, P. J. Chase et al., "Weight loss via diet and exercise improves exercise breathing mechanics in obese men," Chest, vol. 140, no. 2, pp. 454-460, 2011.

[19] N. Miyatake, H. Nishikawa, A. Morishita et al., "Evaluation of exercise prescription for hypertensive obese men by ventilatory threshold," Journal of the Chinese Medical Association, vol. 66, no. 10, pp. 572-578, 2003.

[20] W. Kindermann, G. Simon, and J. Keul, “The significance of the aerobic-anaerobic transition for the determination of work load intensities during endurance training," European Journal 
of Applied Physiology and Occupational Physiology, vol. 42, no. 1, pp. 25-34, 1979.

[21] R. P. Kim, S. V. Edelman, and D. D. Kim, "Musculoskeletal complications of diabetes mellitus," Clinical Diabetes, vol. 19, no. 7, pp. 132-135, 2001.

[22] S. G. DaSilva, L. Guidetti, C. F. Buzzachera et al., "The influence of adiposity on physiological, perceptual, and affective responses during walking at a self-selected pace," Perceptual and Motor Skills, vol. 109, no. 1, pp. 41-60, 2009.

[23] R. D. Morton, D. J. West, J. W. Stephens, S. C. Bain, and R. M. Bracken, "Heart rate prescribed walking training improves cardiorespiratory fitness but not glycaemic control in people with type 2 diabetes," Journal of Sports Sciences, vol. 28, no. 1, pp. 93-99, 2010.

[24] WHO Document Production Services, Report of a WHO/IDF Consultation Definition and Diagnosis of Diabetes Mellitus and Intermediate Hyperglycemia, WHO Document Production Services, Geneva, Switzerland, 2006.

[25] T. Sofková, M. Přidalová, J. Mitáš, and J. Pelclová, “The level of neighborhood walkability in a place of residence and its effect on body composition in obese and overweight women," Central European Journal of Public Health, vol. 21, no. 4, pp. 184-189, 2013.

[26] R. S. Guerra, T. F. Amaral, E. A. Marques, J. Mota, and M. T. Restivo, "Anatomical location for waist circumference measurement in older adults: a preliminary study," Nutricion Hospitalaria, vol. 27, no. 5, pp. 1554-1561, 2012.

[27] B. Balke and R. W. Ware, "An experimental study of physical fitness of Air Force personnel," United States Armed Forces Medical Journal, vol. 10, no. 6, pp. 675-688, 1959.

[28] S. E. Selig, I. Levinger, A. D. Williams et al., "Exercise \& Sports Science Australia Position Statement on exercise training and chronic heart failure," Journal of Science and Medicine in Sport, vol. 13, no. 3, pp. 288-294, 2010.

[29] J. M. Lee, D. R. Bassett Jr., D. L. Thompson, and E. C. Fitzugh, "Validation of the cosmed fitmate for prediction of maximal oxygen consumption," Journal of Strength and Conditioning Research, vol. 25, no. 9, pp. 2573-2579, 2011.

[30] R. D. Hagan and M. G. Smith, "Pulmonary ventilation in relation to oxygen uptake and carbon dioxide production during incremental load work," International Journal of Sports Medicine, vol. 5, no. 4, pp. 193-197, 1984.

[31] C. Baldari and L. Guidetti, " $\mathrm{VO}_{2 \max }$, ventilatory and anaerobic thresholds in rhythmic gymnasts and young female dancers," Journal of Sports Medicine and Physical Fitness, vol. 41, no. 2, pp. 177-182, 2001.

[32] E. L. Rosado, R. S. de Brito, J. Bressan, and J. A. M. Hernández, "Effectiveness of prediction equations in estimating energy expenditure sample of Brazilian and Spanish women with excess body weight," Nutricion Hospitalaria, vol. 29, no. 3, pp. 513-518, 2014.

[33] B. E. Ainsworth, J. Bassett D.R., S. J. Strath et al., "Comparison of three methods for measuring the time spent in physical activity," Medicine and Science in Sports and Exercise, vol. 32, no. 9, pp. S457-S464, 2000.

[34] N. Gordon, “The exercise prescription," in The Health Professional's Guide to Diabetes and Exercise, pp. 71-82, American Diabetes Association, Alexandria, Va, USA, 1995.

[35] D. Umpierre, P. A. B. Ribeiro, C. K. Kramer et al., "Physical activity advice only or structured exercise training and association with $\mathrm{HbAlc}$ levels in type 2 diabetes: a systematic review and meta-analysis," JAMA-Journal of the American Medical Association, vol. 305, no. 17, pp. 1790-1799, 2011.

[36] N. G. Boulé, C. Robert, G. J. Bell et al., "Metformin and exercise in type 2 diabetes: examining treatment modality interactions," Diabetes Care, vol. 34, no. 7, pp. 1469-1474, 2011.

[37] O. P. Adams, "The impact of brief high-intensity exercise on blood glucose levels," Diabetes, Metabolic Syndrome and Obesity, vol. 6, no. 6, pp. 113-122, 2013.

[38] K. Z. Walker, L. S. Piers, R. S. Putt, J. A. Jones, and K. O’Dea, "Effects of regular walking on cardiovascular risk factors and body composition in normoglycemic women and women with type 2 diabetes," Diabetes Care, vol. 22, no. 4, pp. 555-561, 1999.

[39] S. Shenoy, R. Guglani, and J. S. Sandhu, "Effectiveness of an aerobic walking program using heart rate monitor and pedometer on the parameters of diabetes control in Asian Indians with type 2 diabetes," Primary Care Diabetes, vol. 4, no. 1, pp. 41-45, 2010.

[40] E. S. Kilpatrick, "Haemoglobin $\mathrm{A}_{1 c}$ in the diagnosis and monitoring of diabetes mellitus," Journal of Clinical Pathology, vol. 61, no. 9, pp. 977-982, 2008.

[41] A. Makris and G. D. Foster, "Dietary approaches to the treatment of obesity," Psychiatric Clinics of North America, vol. 34, no. 4, pp. 813-827, 2011.

[42] M. L. Dansinger, J. A. Gleason, J. L. Griffith, H. P. Selker, and E. J. Schaefer, "Comparison of the Atkins, Ornish, Weight Watchers, and Zone Diets for weight loss and heart disease risk reduction: a randomized trial," Journal of the American Medical Association, vol. 293, no. 1, pp. 43-53, 2005.

[43] J. M. Jakicic, B. H. Marcus, K. I. Gallagher, M. Napolitano, and W. Lang, "Effect of exercise duration and intensity on weight loss in overweight, sedentary women: a randomized trial," Journal of the American Medical Association, vol. 290, no. 10, pp. 1323-1330, 2003.

[44] P. Ekkekakis and E. Lind, "Exercise does not feel the same when you are overweight: the impact of self-selected and imposed intensity on affect and exertion," International Journal of Obesity, vol. 30, no. 4, pp. 652-660, 2006.

[45] S. G. Dasilva, L. Guidetti, C. F. Buzzachera et al., "Gender-based differences in substrate use during exercise at a self-selected pace," Journal of Strength and Conditioning Research, vol. 25, no. 9, pp. 2544-2551, 2011.

[46] J. Larose, R. J. Sigal, N. G. Boulé et al., "Effect of exercise training on physical fitness in type II diabetes mellitus," Medicine and Science in Sports and Exercise, vol. 42, no. 8, pp. 1439-1447, 2010. 


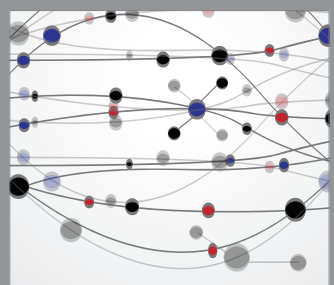

The Scientific World Journal
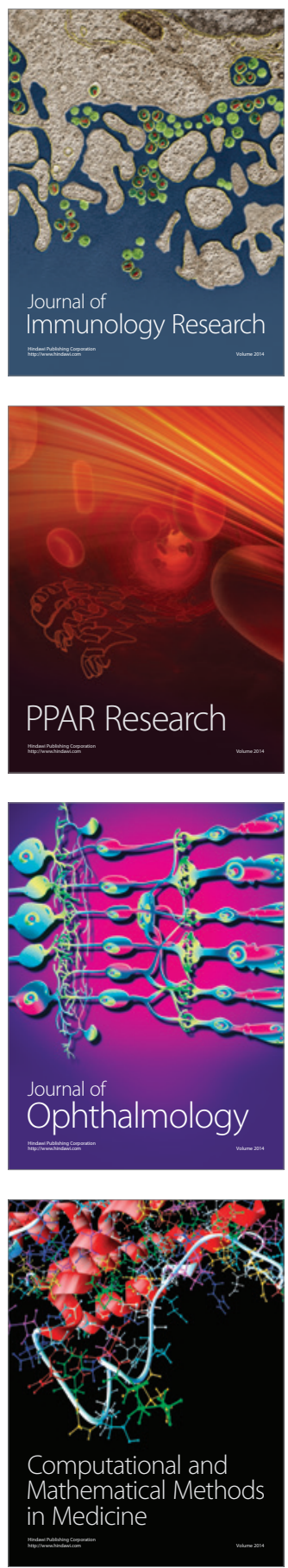

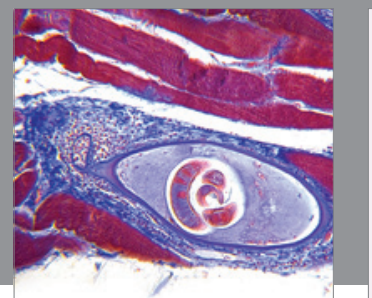

Gastroenterology

Research and Practice
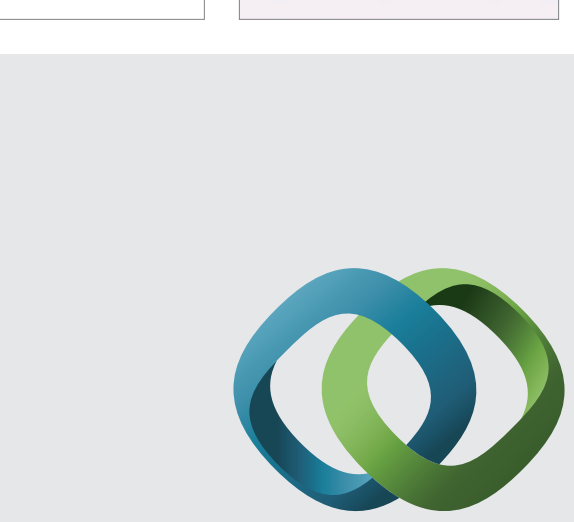

\section{Hindawi}

Submit your manuscripts at

http://www.hindawi.com
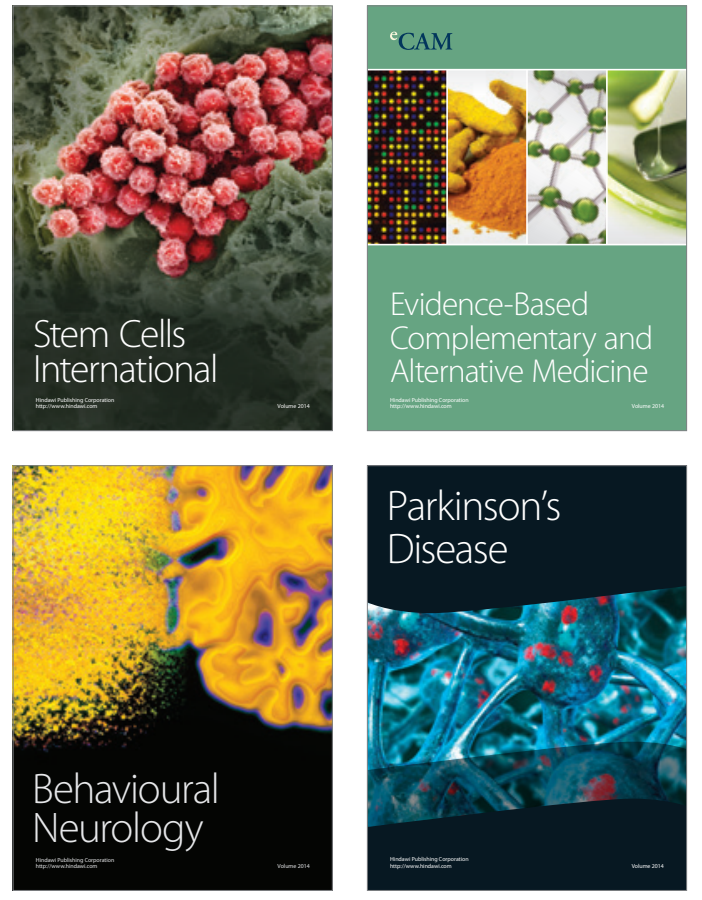
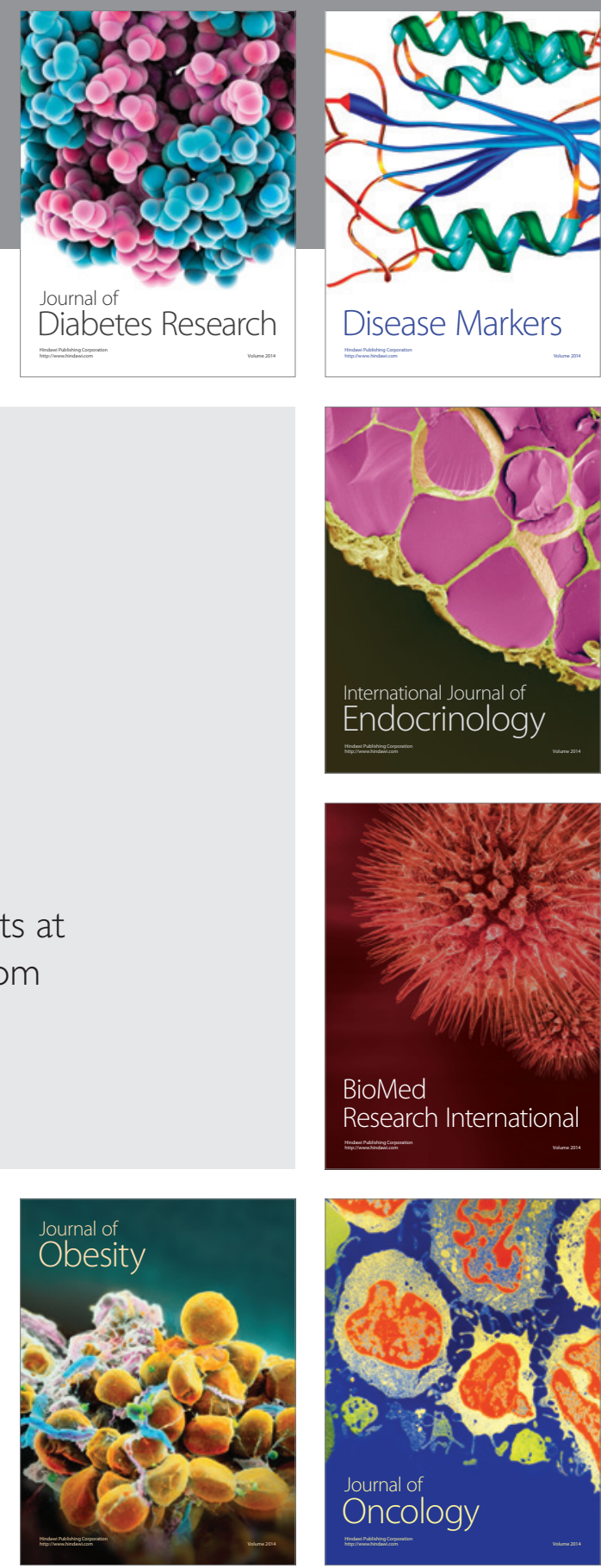

Disease Markers
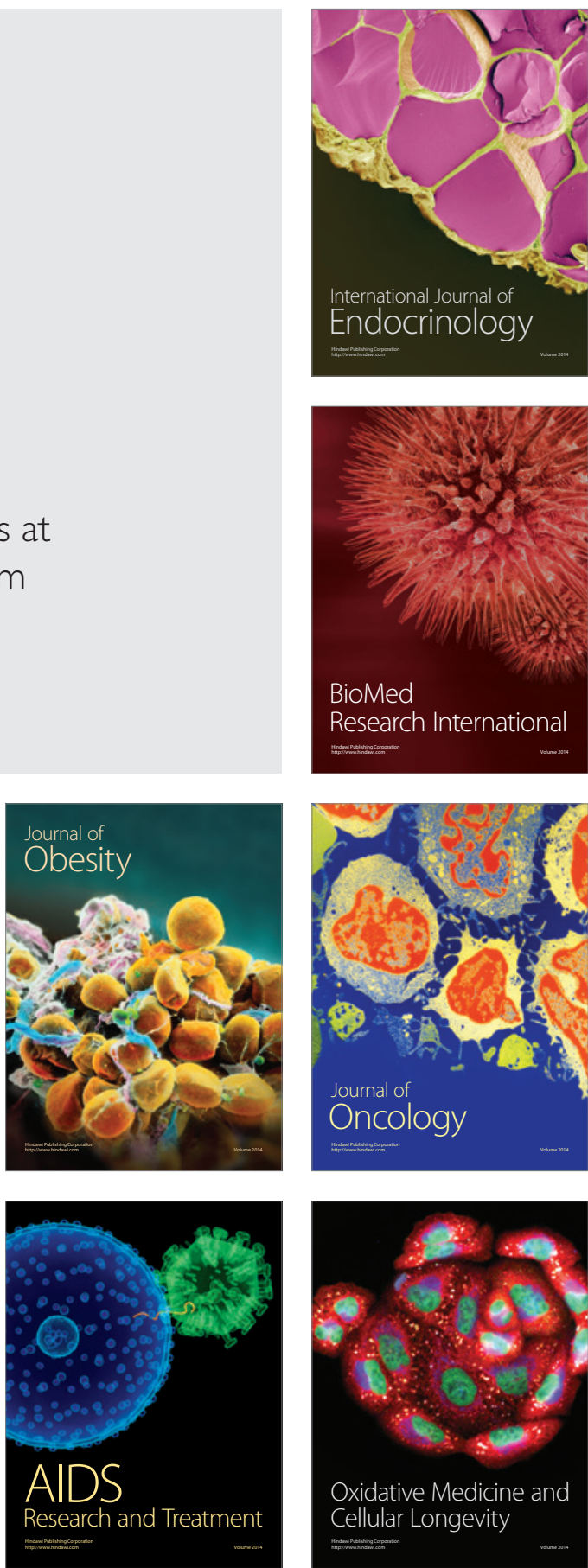